\title{
Influence of Machine Translation on the Online Cross-cultural Communication
}

\author{
Ming Lu \\ School of Foreign Languages \\ Wuhan University of Science and Technology \\ Wuhan, China
}

\author{
Sile Niu \\ School of Foreign Languages \\ Wuhan University of Science and Technology \\ Wuhan, China
}

\begin{abstract}
The paper studies the relationship and interactions between machine translation (MT) and intercultural communication online. The analyses reveal the positive and negative influences of MT on the online cross-cultural communication and give the possible suggestion to the further development.
\end{abstract}

Keywords-machine translation; online cross-cultural communication; MT-embedded applications

\section{INTRODUCTION}

Machine Translation (MT), or we called Automatic/Artificial Computer Translation, is becoming one of the most popular technology in computer science which is used extensively in the bursting internet cross-cultural communication, and served both in academic fields and daily civilian uses. Generally, this kind of machine-aided translation could be defined as "the attempt to automate all or part of the process of translating from one human language to another" (Arnold, 1995: 12). MT is also a sub-field of computational linguistics that investigates the use of computer software to perform instant interpretations. At its basic level, MT performs "simple substitution of words in one natural language for words in another" (Hutchins, 1992: 2), setting modifications through programmed MT rationales.

However, on the other hand, MT takes a significant part in the internet cross-cultural communication and plays a key role in the modern computer applications. The MT software can provide auto-selected page interfaces and real-time translations to the internet beginners. From this aspect we could find some characteristics in the online cross-cultural communication which distinguish itself from any other types of cross-cultural communications: Firstly, the "electronic interactions differ significantly from face-to-face exchange." and "it increases our range of human connectedness and the number of ways in which we are able to make contact with others" (Harasim, 1993 17). Next, there is a significant interaction between culture, information, and personal online communication in the network. "The internet provides another context and channel for people to meet with strangers for the first time, initiate meaningful and satisfying conversations, and build stable, long-term relationships" (Yung, 2005: 117). But "how the cultural groups or individuals may differ in their perceptions

The paper is sponsored by Wuhan University of Science and Technology (Project No.: 2013xz039). about their preferred communication behavior" (Kim, 2006: 129) and accomplish a successful cross-cultural communication online? Moreover, this virtualized social intercourses are more abstract to the cross-cultural communicators, their actions may vary from the established patterns of behavior in the actual life. Cultural shocks and collisions on the internet are more difficult to explain due to the language barrier and the simulated communicative interfaces. So under this situation, the computer-mediated systems lead by machine translation software will take their effects in sorting the multi-cultural resources on the internet. During the whole process the use of MT will make crucial effects to the results of the online intercommunications.

\section{MACHINE TRANSLATION IN THE ONLINE COMMUNICATION}

\section{A. Interactions between MT and Online Communication}

From Eugene Nida's book "Language Culture and Translating", the mentality designing concepts of automatic interpretation and the needs of the unmanned translation could be discovered as "the drive of substitutions" (Nida, 1993: 36). He describes the relationships between people and the crosscultural translation work as a "dynamic equivalence approach" (Nida, 1993: 27) that applies to the textual delivered messages. Some basic machine translation tools today still follow the traditional routine of the "simple substitution of words" with the mechanized dynamical database. Normally, by using the net translators, most interpretations are based on a lexical conceptual structure. And the whole interpreting process are all being held in a MT plug-in module to achieve the instantaneous translation. The word-to-word translation is the marked feature (Quirk, 2004).

The MSNShell (Microsoft Service Network messenger assistant plug-in) as an example: This is a typical paradigm of how the MT software is embedded into an online communication tool. When the user opens a contact window and starts an online communication, there are at least two types of machine translation plug-in for him to choose. One is the MSNShell Translate Assistant (TA). The TA provides two kinds of automatic translation aids - one is the "pasting \& copy" assistance, and the other is the traditional dictionary-like word paraphrases. Besides TA, the IM(instant messengers) itself also has an MT tool, the MSN Live Translator(also called the ICS-Live translation robot). This software was implanted as 
one of your messenger contact, and the translator codes will be automatically detected when you typing any messages to this contact. The translation results will be sent back in the forms of instant textual messages.

There is a large language-independent portion of the MT system - a self-dependent device which is exactly same to the internet search engine system, "a kind of ' $\mathrm{X}$-to-Y translation shell,' making it easier to handle new language pairs" (McCord, 1999: 4). This suggests that the scale of MT system is actually confined in the separated rules of transfer steps. By eliminating or adding the parts of the unnecessary language aiding groupware, the MT system will keep its unity of the translating functions and the consideration of the incorrect MT-to-AC (automatic catch) manipulation could be excluded from the core devices of the independent web pages.

Much of the internet users would simply ignore the setups of the language rendering aids, but the leading trends of the internet MT practices are growing. The rudiment of the computer-mediated translation services is changing the way of online cross-cultural communication by providing the new automatic translation experiences.

\section{B. MT and Online Communication on Web Pages}

The web online environment is a series of intercultural linked communicative pages. Firstly, the website allows users to do more than just retrieve information, encapsulates the idea of the proliferation of inter-connectivity and interactivity of web-delivered content. It is a set of collective online information that forms the basis of the web-a programmable web page characterized by the user participation, openness, and network effects under a cross-cultural background. Those patterns and characteristics of web system highly require the auto-mediated language translation system to meet the demands of the worldwide internet users. The integrated language automation of the web practices is pushing the MT applications to interfere the cross-cultural communication on the internet. Without the bases of the user-oriented web pages, the influences of MT on the online intercultural communication will turned back to the traditional computer rule-based phase.

Recent years we have witnessed the lasting momentous advances in machine assisted translation and the bursting cultural diversity on the internet. On one hand, the internet is undertaking in the rapid growth of online cross-cultural communication requests. There is an everlasting need for automatic translation. On the other hand, the internet has also emerged as the ultimate sources for information, language, and translation. The "automatization" is ascending to the top of the new prototypes which containing the next-generation online communication with the use of MT technology and its further development (Jason, 2006). Here the paper will try to give an example of how the MT interferes with the web communication and figure out the major features of the whole internet social intercourse.

In Google Creative Commons, the three major groups of GCC are:

- http://www.google.com/advanced_search?hl=en

- http://www.google.com/ig

\section{- http://creativecommons.org/}

This is a typical web online searching system designed for person-to-person communications. In this case, the net users do not need to concern the existence of the MT threading process when they are surfing on the Google sites, the whole MT navigation will start automatically to the web browsers. Without any terminal behaviors, the site will detect the endpoint of the user's computing system and the net preference. In another word, for different online IP addresses and the default sets of the OS (Operating Systems), the automatically selected user interface will be provided beforehand. Therefore, the machine translation will be added in the upcoming sites, including the language we see and the content we are going to express on the web interactional visages. If the user is not satisfied with the automatic translation results, he could choose to the web-implanted language tools to start a more professional and complete MT-aided translation. All the crosscultural communications start at the moment the user begin searching their wanted information on the internet.

So here are the questions about this cross-cultural activity online: what exactly did the machine translation results affect the quality of the net cross-cultural information exchange? To analyze the influences of the translation work in detail, we could carve up those effects to two groups: the positive influences and negative influences of MT to the internet crosscultural communication.

\section{POSITIVE INFLUENCE OF MT ON THE ONLINE CROSS- CULTURAL COMMUNICATION}

It is clear to see that by using the machine translation system the roominess of the internet cross-cultural communication are impelled to a vast range. The machine translation system enables the end users to save a considerable amount of time in finding their adaptable communication interfaces. In another word, this system confined the boundaries and boarders on the internet communicative platforms so the online socializing activities could lock their interested fields in a more simple way. The internet interlocutors from different cultural backgrounds now share a universal platform of communication. The following will try to analyze the positively brought by the MT system on the internet.

\section{A. Universality of MT for Fast Online Messaging}

By equipping with automatic languages navigation systems, the net users do have the chance to share the universality of the online cross-cultural communicative system. The software and websites are accompanied by a "raft of affordances that expand how we teach, communicate, learn and create knowledge" (McLoughlin, 2008: 12). Same to the development of the new MT implanting technology, new trends are emerging in the way how the information is distributed and received. The bursting web services such as blogs, wikis and social book marking applications, as well as social networking sites like Twitter and Facebook are seen as the personal virtual communities. Based on the "microcontents" i.e., the digital content in small fragments that combined individuals to produce new patterns, images and culture interpretations. Under such online environments, the universality of MT- 
mediated online communications includes the quickness of the net information exchange system, the exactness of the instant translation and the referential information feedbacks.

The universality of MT is not only presented in the time scales and the deepness of the internet resources. Automated translation tools have been around for a long time, and new techniques are boosting their performance, the key feature of its performances in the online communication practices is the coordination of the translated materials. Taking GCC and YouTube.com as examples, we could notice that the interfaces of those web sites are highly mediated by an automatic language aiding system. The functional buttons or links on the web interfaces, whether graphical or textual, are all being neutralized to the user's preferences based on their behaviors of the formal messaging actions on the site. These data-driven systems "learn" what makes a good translation and what doesn't, and then they use the probability and the data statistics of the MT mediating system to perform a close translation result (McCord, 1999: 20). This translation process is very universal to the MT users with different cultural backgrounds, what they could share is the similar web interfaces that present in their mother languages and personal viewing preferences. The new direction in the MT language navigation is to see how the internet communication participants can affect the autoselected online communicative interfaces. The mixed systems which combined the translation memory databases and the lexicalized machine translation software are optimized to the online web interfaces, helping the users to share an open crosscultural platform online. In essence, the universality in online cross-cultural messaging suggests the advantages of the "invisible" language mediation on the internet, thousands of the net socializing partakers with different mother tongues and cultural backgrounds could share a trans-civilization social intercourse on the same web site.

\section{B. Convenience of the Embedded MT Application}

The characteristic of the embedded MT application, or the so called "the embeddability" of the MT software (the plug-in system) is another way to the online cross-cultural communication, it positively supported the instant online messages in a computational favorable way.

The combination of MT and the net socializing media is an interactive approach to the elimination of online manual MT applications. Despite the growing trend of the online interactive medium, there would appear to be "a lack of comprehensive research methodology for evaluating the degree of standardization in multinational corporations' online communication strategies across differing cultures" (Rivas, 2002: 36). However, to construct a research framework for cross-cultural comparison of user collaborated web pages, the partitioned MT-aided online communication must be built. As we mentioned before, the current trends of internet crosscultural communication are promoted to the automatic language navigation interfaces. Demands have been increasing for internal machine translation systems to handle a wider range of different languages and internet cross-cultural domains. Embedding the MT auto-detect system will not only benefit the integrated MT applications of the online communicative interfaces, but enable the users to characterize their own communication instruments. The post-editing efforts in this MT system are rare in the general practices, now the external links of the cross-cultural online communication are minified to the branches of the web menu. In the case of GCC online cross-cultural communication, it means the searching result will always be collocated according to the users' preferences like their default language and the advanced searching configurations. This positive effect could be described as a permanent language service in the whole online cross-cultural communication system - the integration of the automatic translation and the web-based network.

\section{NEGATIVE INFLUENCE AND POSSIBLE AIDS}

In a subject-oriented view of MT, the whole system also bears a lot of troubles in actual online cross-cultural practices. The two questions should be noticed are: the unavoidable computational miscalculations of MT and the user's efficiency in communicative MT presentations. The most severe miscalculation caused by MT in the online cross-cultural communications is the web resource tagging system. And a poor user's configuration to the MT software could make a series of translation mistakes in operating the online translation services.

\section{A. MT Failure in Labeling the Web Resources}

A computer translation system needs a capability to make the terms of MT knowledge process being held in the unmanned status (Hutchins, 1992: 63). Knowledge processing means a computer takes over thought and judgment of humans using knowledge of the collectivity. Machine translation systems can deal with knowledge and contents in their databases, but their representation of knowledge or information is different from the way of man-made documentary archives. Moreover, their representations are language dependent by the digital structure of the computer system. The primitive MT system used to represent information independently. On the actual online communication cases, the web interfaces we have met with the automatic translating devices are firmly set. Less "space" is obligated to the online communicative platforms.

To reinforce the MT information collecting ability, the tagging system is proposed to assist the users in their online cross-cultural activities. So the MT tagging system could be a particular case to state the negative influence of the machine translated web pages in an online cross-cultural activity. "MT Tagging" refers to a "massified body of hand-labeled data which are used to make with the aid of a finite state recognizer in MT assisted cross-cultural communication" (Hutchins, 2005: 7). To be more specific, it refers to a man added electronic label to state one's shared information online. By establishing this system the MT searching engine could improve its efficiency in matching the dynamic controlled information resources, so the communicators from all kinds of cultural backgrounds could be matched through a minor reserved word groups. From the former studies of this paper, we could see that the automatic language navigation applying to the web interfaces is basically a downsizing process to the plurilingual communicative designs. To get the universality and the quickness of machine translation, the computer system and the users have no choice or option but to reduce the information quantity by providing less message bits to the MT software. 
This special feature is propitious to web sites because it keeps the conciseness of the intercultural interfaces on the web page, making those webs have neat surroundings with fast link speed. It promotes the efficiency of the intercultural online activities. But it brings a series of troubles to the net users - taking the former GCC based online cross-cultural communicative activities as examples, when a net user is trying to share some of his textual or graphical document with his net friends all over the world on GCC, the first step for him is to tag all his contents with a short name label using his native language and submit them to the tag ranking list of the web site. From this step the MT system will automatically translate the custommade tags to the supported languages so the rest of the net users who use different languages could identify the documents in their own web links. Since the E-Labels themselves do not need to keep a physic form of limitation (Liu Zhengdong, 2001: 5-6), the users could not define the contents of the label by the information details they sent to the searching engine, and all the OS supported languages and interpunctions could be used in the annotations. By accepting the input texts of the labels, the MT system will take part in the arrangement of the electronic tag cards, especially the embedded MT components of the system. Although the integrated auto-language navigation system could precisely match the possible userlanguage preference according to the previous multicultural online experiencing investigations, the lack of the customeroriented information collectorship will affect some personalized tags which are likely to fail the MT language mediating mechanism. This minor flaw of the MT could cause a series of troubles in a foreign information exchanging activity.

Since the tagging system could not discern the differences between an "information annotation" and a computer-mediated monitor message, the links of the network and intercultural communication were closed due to a machine logical error (Wang Shaochun, 2001: 6). Therefore the online cross-cultural communication will fall into a dilemma: to alter the searching directions like posting a static thread on the traditional internet bug report or end the request in order to avoid false information provided by the translation system. This dilemma will damage the quality of the online intercultural contacts severely and stuff a jet lag to the real time online communication.

\section{B. Misinterpretations Caused by Invalid User Settings}

The general MT setting of the user's communication tool is very important to the quality of the cross-cultural interactions online. Users need to learn how to configure the MT settings manually so the functions of the language aids could be fully unfolded. On the computer side, all the functions of the MT aids need to be enabled by the client permission so the automatic translation could begin their work at the real time web circumstances. On the user's side, the setting could help him to keep his personalized software updated with the latest MT databases and to open the access of public information share. The internet cross-cultural communication is much to a collaborative work, the partnership is essential for a successful online interaction. By finding the shortcomings of this virtual group we could also fish out the respond of the online computational cross-cultural environment and make improvements to the MT-aided internet web socializing activities.
For GCC and IM software, the two major intercultural communication platforms online, the MT settings and the language controls are integrated to the main options of the communicative interfaces. Basically, invalid user configurations have two features: the restrictions of the textual translation and the subscriptions of the alternative automatic translation comment margins (Rahul, 2004: 11). When the users open the applications, those two patterns of settings will start the translation aids with the embedded MT applications, and the automatic translation check will begin the scanning process to the online communicative web interfaces. The users could change the setting during their operations on the internet, but it will also change the implantation of the automatic translating system, therefore the instant messages will be redirected to the electronically transferred character codes online. So to examine the way how the incorrect user settings will interfere the simultaneous interpretations online, we should check the user's translation request before they start configuring their software.

Current machine translation software often allows for customization by domain or profession (such as foreign language teaching or computer science) like Lingoes Online, Babylon Professional Edition or Trados, for a long-term cooperative customer, these software will provide a private account to the user so they can save their translation preferences in their mail boxes or software settings. Those personal settings will take many strong effects to the user's online cross-cultural communications. Unlike the automatic MT tagging system, the setting in the user's private account is firmly established according to the general principles of MT categories and is optional to the customer. Details of these configurations include the static textbox for monolingual input and advanced real time translations (Rivas, 2002: 41). The default restriction in the software of the translating textbox is to clog the user's native language input within limited characters and words, nor to extend the length of a given text. Some net users are always confused by this concept: this default restriction of textual contents seems unavailable to the paragraphed messages, and the translation results could not convert to the readable computer language aids. The communicators will have much difficulties in catching up with each other by the unidentified machine translation works, and the communications online would be interrupted due to the incorrect software settings.

Another negative practice to the MT-aided online communication is the violation of the alternative MT information feedback (Rahul, 2004: 16). This problem often occurs in the IM based online communications. In order to get a faster translation, the users always rely too much on the instant alternative MT system like the MSN Live Translator or GCC's automatic web translation. The machine translators themselves were well developed to meet the requirements of the instant computational messages online. Normally they will provide some referential information to the translated contents like the explanation of the proper nouns and special language features like the tips of MT selections. However, the users would like to quote that alternative information in their actual online communications. This is the reason why the most web sites disabled their individual information broadcast function 
online (McLoughlin, 2008: 22). The message that submitted by the users contains a lot of referential information created by the MT software. This information is irrelevant to the crosscultural communication and will disturb the fluency of the online instant messages while the users are hoping to make their mails or letters to be translated in a more concise way.

\section{Possible Aids to the Negative Effect}

Although the current MT-based international communication still faces a lot of troubles both from the technical terms and the user's operations, we can always bring some helpful solutions.

1) Manual MT data updates:Based on the frequency of the online automatic language utilization and the constant MT request on the internet, the manual adjustment to the MT inputs could take some positive effect to guide the computer for better language navigation during its pre-processes. An enhanced operation in changing the requested messages could improve the efficiency of the MT system in web pages. For example, when a foreign language teacher from China is using the language tools provided by GCC to upload his academic research papers on the internet, he could also upload some new MT processed materials like the auto statistics data from his MT account. The MT system from the web site then will automatically sort the status of his papers to the public information collectors online, so other foreign language teachers from different countries could rapidly locate his latest archives on the same platform with the aids of the MT navigation services. Both the information providers and the receiver on the internet could step over the barriers of the boundless databases on the internet. Academic resources could be reached in another convenient way.

Unlike the superfluous machine translation tags or information labels, by providing one's own "informational RNA" via the computer mediated language translation system, the messages extract from the users' prompt online status could avoid the redundant information blocks on the multi-cultural environment (Jason, 2006: 11). In the former web sites, users are always asked to fill the personal information passively in order to get the access of internet resources. But if they could add more updates of his MT software status and translated messages through the online public broadcasts, more and more communicators could discover his papers directly by only a few steps. Less time and energy will be saved due to the daily manual updated information materials.

This method recasts the source applications of the MT system, and users need to understand that they are not only uploading his documents to other people online, but also providing a language filter to help the net users to locate his works. So this time the machine translator could also be operated like a cross-cultural communicator on the internet, it is a new way to develop the mutual benefits of the internet users.

2) Multi-sample picks of the original text: This is a conventional method in adjusting the machine translation results, but we can also add a few more steps to this procedure so it can deal with the new MT system. To translate a given text by MT software, we have several options: to put the raw material into a formatted text file and send the file to the MT applications (Quirk, 2004: 7), to start the language scanning function of MT software so the system could generate its own translating balances to the original copy(like Trados Professional, a MT software), or to conform the given text onto the web site so the automatic language navigation could identify the contents and provide synchronous translation matches (Wang Shaochun, 2001: 4). By observing the different ways and functions of MT applications, we could figure out the basic patterns of the manual preparation work from the user's side, and a series of revises would be done before the prepared information are sent to the MT processing system.

From the basic principles of the MT layouts, the users could split the text to smaller samples to adapt different forms of MT interfaces. Taking Lingoes Online as an example, when the users install this software onto his computer and configure it to aid his IM software as a language navigator, he could get four types of MT input interfaces, they are the authoritative online dictionaries with full word definitions, the multilingual web-based encyclopedia, the lexical database searching engine and the instant web translation tool. Each interface accepts programmed text to perform an automatic language analysis. To improve the accuracy of the translation results, the user could firstly copy their given text into the searching engine to seek matched materials for the latter translation, and then the results from the online information searcher will be exported to the main menu of the software. The second step to the user is to pick interlinks of the matched information to the web analysis. This technique is particularly effective in domains where the formal or formulaic language is used. It follows the coded MT documents continued to produce the practical outputs. They are more precise and reliable than the lexicon translated material or less standardized MT works. Finally the users could handle the translated results to be updated by the MT software with the information collector. So his contacts could soon locate his new status by the same language tools. For the communicator on the other side, they could read the MT converted file with the backup language advisor like the online dictionaries and encyclopedia to get the detail instructions of the translated text. In other words, the origins of the machine translation could be traced by the user. It will bring many conveniences to the cross-cultural communicators online.

According the different functions of the online MT system, the users could make some optimization to the whole automatic translation process by picking all kinds of information interlink to the software. Once the user is fluent with this skill, he could enjoy a better online cross-cultural communication environment within his own knowledge scope.

\section{CONCLUSION}

The aim of using computers for translation is not to "emulate or rival human translation" but to produce "rough translations which can serve as drafts for online published translations", as means for accessing foreign-language information and cross-language communication aids (Hutchins, 2006: 14). The field of machine translation covers the usage, 
research and development of computer aids and systems, ranging from production systems for large corporations to language aids for individuals. Automatic translation of web sites is growing in the intercultural online communication practices.

Although most MT researchers are still aiming for autonomous translation systems, where human information has to be updated regularly and intervention is minimal (Hutchins, 2006: 5), there are also many researches of dialogue-based and computer-interactive systems, including the use of controlled or "regularized" input with the aim of ensured higher quality outputs. The future applicable MT system will take its unique places both in the internet communication and the online computer practices. By the perfection of the internet, the crosscultural communication online and the MT tools will get a more intimate relationship to people's life. The influences will last long to the world wide intercultural communicative activities.

\section{REFERENCES}

[1] Arnold, Doug. Machine Translation: An Introductory Guide [M]. Essex: University of Essex Press, 1995.

[2] Harasim, Linda M. Global Networks: Computers and International Communication [M]. Massachusetts: MIT Press, 1993.

[3] Hutchins, John. An Introduction to Machine Translation [M]. London: London Academic Press. 1992.

[4] Hutchins, John. The History of Machine Translation [M]. Oxford: Pergamon Press. 2005.

[5] Hutchins, John. Machine Translation in Europe and North America: Brief Account of Current Status and Future Prospects [M]. Oxford: Pergamon Press. 2006

[6] Jason, S. Chang. Machine Translation Fast Forward [M]. Taipei : National Tsing Hua University, 2006.

[7] Kim, Mintson. Cross-cultural comparisons of the perceived importance of conversational constraints [J]. Human Communication Research, 2006(21): 128-151.

[8] McCord, Michael. A New version of the machine translation system [J]. Literary and Linguistic Computing, 1999(3): 4-18.

[9] McLoughlin, Catherine. Social Software and Participatory Learning: Technology affordances in the Web 2.0 era [M]. Melbourne: Australian Catholic University Press, 2008.

[10] Nida, Eugene. Language Culture and Translating [M]. Shanghai : Shanghai Foreign Language Education Press. 1993.

[11] Quirk, Chris \& Chris Brockett, William Dolan. Monolingual Machine Translation for Paraphrase Generation [M]. Arkansas : Microsoft Research Facility, Conway Press, 2004.

[12] Rahul, Nair \& Kedar Shiroor. BuzzTrans: Evaluating a Translating Instant Messenger Client [M]. Atlanta: Georgia Institute of Technology, 2004.

[13] Rivas, Javier Alonso \& Shintaro Okazaki. A content analysis of multinationals' web communication strategies: cross-cultural research framework and pre-testing [J]. Journal: Internet Research, 2002(12): 3641.

[14] Yung, Yan Ono \& Hara, K. Computer-mediated relationship development: a cross-cultural comparison [J]. Journal of ComputerMediated Communication, 2005(11):117-128.

[15] Liu Zhengdong, Zhang Bingquan. Real-time search translation site search engine agent technology $[\mathrm{M}]$ Shenyang: Shenyang Institute of Technology Press, 2001.

[16] Wang Shaochun, Chen Jiajun, Wang Qixiang, Xie Junyuan. Internet Online Translation Browser Technology [J], Computer Application Research, 2001 (18): 22-37. 